racin,Cloxacillinand pipracillin.resistantto other antibiotics were: Sulfametoxazole 92\%, Nalidixic acid 53\%, Ampicillin 89\%, Nitrafurantoin 9\%, Cephotaxime 55.3\%, Cefixime67\%, Gentamicin72\% ,Cephalexin $75.6 \%$, Ciprofloxacin $17.5 \%$

Conclusion: Periodic review and formulation of antiobiotic policy are needed for control of acquisition of drug resistance. Further studies on better understanding of interaction of different virulence factors at molecular level are necessary as most urovirulent strain express multiple virulence factors simultaneously.

Key words : Uropathogenic E.coli ,Antibiotic susceptibility pattern,UTI

1397

\section{DETECTION OF RESISTANCE TO CARBAPENEMS IN E.COLI AND KLEBSIELLA PNEUMONIA FROM URINE OF CHILDREN WITH UTI IN MOFEED CHILDREN HOSPITAL}

A. Sanaei, A. Karimi, M. Malekan, F. Fallah, M. Navidinia, L. Akhoundtabar

Shahid Beheshti University of Medical Sciences, Pediatric Infectious Disease Research Center, Tehran, Iran

Introduction and objectives: infection by Enterobacteriaceae which are resistant to carbapenems is challenging problem in health care centers. The correct monitoring of patients for treatment by carbapenems was one of the most significant considering in this study.

Methods: the urine samples of patients were gathered during six months. E.coli and Klebsiellae strains were detected. Susceptibility testings such determining of $\mathrm{MIC}$ and agar diffusion agar were performed for meropenem and imipenem for these strains and then carbapenemase coding gene detected by PCR method.

Results : 197 strains isolated fro urine samples in which $138 \mathrm{E}$.coli and 58 Klebsiella pneumoniae were detected . 5 strains of E.coli ( $3.6 \%$ ) and 3 strains of Klebsiella ( $5.1 \%$ ) were resistant to Imipenem and Meropenem and then kpc2 and kpc3 genes of their resistance were detected by PCR.

Conclusion: there is no any comprehensive program for screening of resistant strains of bacteria to carbapenems in our country and worldwide but epidemiologic increasing of these strains should be considered.
Key words: carbapenemase - E.coli - Klebsiella pneumoniae

\section{8}

\section{HAEMATOLOGICAL, C-REACTIVE PROTEIN AND PROCALCITONIN BEHAVIOUR IN NEONATAL SEPSIS}

\author{
C. Almeida ${ }^{1}$, M. Pinto ${ }^{2}$, S. Ferreira ${ }^{2}$, D. Schmith ${ }^{2}$, \\ J. Garrido ${ }^{2}$, M. Carrapato ${ }^{1,2}$ \\ ${ }^{1}$ Fernando Pessoa University, Oporto, ${ }^{2}$ São \\ Sebastião Hospital, Santa Maria da Feira, Portugal
}

Introduction: Neonatal sepsis remains a clinical diagnostic problem. This study analyses haematological, C-reactive protein (CRP) and procalcitonin (PCT) behaviour in different clinical settings of sepsis.

Methodology: Data from neonates diagnosed with sepsis were collected and analysed. PCT determined by VIDAS $^{\circledR}$ BRAHMS PCT, CRP by Immunoturbidimetry and blood counts by Flow Cytometry.

Results: Out of 21 babies, 3 had positive blood cultures and, at the time of diagnosis, PCT was extremely high (64.68, 299.9 and $382.6 \mathrm{ng} / \mathrm{mL}$; reference range: $0.05-5.89 \mathrm{ng} / \mathrm{mL}$ ) and were thrombocytopaenic. Leucocytes and neutrophiles were normal and CRP, with one exception, remained normal. Following antibiotics, PCT declined mirroring improved clinical course whilst the other parameters were generally uninformative. Ten babies with RDS and diagnosed sepsis were reviewed blindly and classified as unlikely; PCT remained either negative or increased slightly. The other parameters varied widely. Seven neonates with clinically compatible sepsis all showed raised PCT whilst the other parameters were indifferently normal or altered. One baby of 33 weeks GA deceased after severe RDS, thought to be infected, with all parameters altered, showed at post-morten severe HMD only.

Conclusion: PCT was shown to be an early marker of confirmed sepsis and follow clinical course. When PCT remained negative or slightly raised sepsis was an unlikely diagnosis. In all those cases where sepsis was a likely possibility, although not confirmed, PCT was also considerably raised. PCT, in general, was a better indicator of sepsis present or absent. However, PCT may be altered in other situation and this needs investigating. 